\title{
Endocrine regulation of the reproductive arrest in the long-winged females of a flightless bug, Pyrrhocoris apterus (Heteroptera: Pyrrhocoridae)
}

\author{
Magdalena HODKOVÁ and Radomír SOCHA \\ Institute of Entomology, Czech Academy of Sciences, Branišovská 31, CZ-370 05 České Budějovice, Czech Republic; \\ e-mail:magda@entu.cas.cz
}

Key words. Pyrrhocoris apterus, macroptery, brachyptery, allatectomy, nervi-allati transection, corpus allatum, terminal oocyte, oviposition

\begin{abstract}
Mechanisms of the suppression of gonadotropic activity of the corpus allatum (CA) in macropterous females were compared with those previously reported for either diapause or starving non-diapause brachypterous females by reciprocal transplantations of the neuroendocrine complexes (comprising the brain-suboesophageal ganglion-corpora cardiaca-CA). The denervated CA stimulated reproduction in most females of all experimental groups suggesting an inhibition of the CA via nervous connections with the brain. The inhibition of the CA within the transplanted neuroendocrine complex was measured by the reproductive performance of feeding recipient females deprived of their own CA. The complex from starving non-diapause brachypterous females stimulated reproduction in $58.3-78.9 \%$ of recipients suggesting that the inhibition of the CA was mostly overcome by the stimulating internal milieu of feeding females. In contrast, the "macropterous" complex stimulated reproduction in only $18.8-37.5 \%$ of recipients, similar to the "diapause brachypterous" complex (32.0\%). The results indicate that the "macropterism", similar to the diapause, is associated with a considerably lower responsiveness of the neuroendocrine complex to humoral stimulation by feeding compared to the responsivenes of the "starving" complex from brachypterous non-diapause females. On the other hand, the CA of macropterous females is of intermediate size between that of the feeding non-diapause and diapause brachypterous females, similar to the CA of the non-diapause brachypterous females deprived of food. Overall, the data suggest that the suppression of the CA activity results from a combination of the diapause-like refractoriness of the neuroendocrine complex with the starvation-like inhibition of the CA growth. Regulation of the CA activity is discussed in relation to the "oogenesis-flight syndrome" recorded for flying wingpolymorphic species of insects.
\end{abstract}

\section{INTRODUCTION}

The firebug, Pyrrhocoris apterus (L.), has two wing morphs: long-winged (macropterous) and short-winged (brachypterous) (Socha, 1993). Earlier studies on brachypterous females demonstrated that reproduction is regulated by photoperiod and food intake. Short-day photoperiod of less than $16 \mathrm{~h}$ light induces facultative reproductive diapause and ovaries remain in pre-vitellogenic stage (Hodek, 1968; Saunders, 1983). Vitellogenesis is also inhibited under long days in the absence of food (Hodková, 1982). In both long-day starving and short-day diapause females, the activity of the corpus allatum (CA) is inhibited via nervous connections with the pars intercerebralis of the brain, but the inhibitory mechanisms are different (Hodková, 1976, 1977a,b,c, 1979, 1982, 1992; Hodková et al., 2001). Although short-day females intensively feed during the first week after adult ecdysis (Šula et al., 1998), the inhibition of their CA is maintained. On the other hand, in females starving under long days, the inhibition of the CA is easily overcome by feeding (Hodková, 1982). In long-day females, the stimulation by feeding can be transmitted to the transplanted neuroendocrine complex of brain-suboesophageal ganglion-corpora cardiaca-corpus allatum (BR-SG-CC-CA) via the hemolymph (Hodková, 1982). Reciprocal transplantations of neuroendocrine complexes between long-day and short-day females of $P$. apterus confirmed the refractoriness of the short-day complex to stimulating internal milieu of feeding recipients (Hodková, 1992). The refractoriness of the short-day complex to feeding seems to be associated with stronger inhibition of the CA compared to the "starving" long-day complex. Different levels of the CA inhibition are indicated by more advanced growth of pre-vitellogenic oocytes in starving long-day females than in short-day females. This difference is most probably caused by a higher activity of the CA in the former case; the ovaries of allatectomized females have "diapause" appearance irrespective of food conditions (Hodková, 1999). Furthermore, the CA of short-day females is smaller compared to starving long-day females (Hodková, 1999).

In macropterous females of $P$. apterus, a temporal reproductive arrest occurs even under long days in the presence of food (Honěk, 1985; Socha \& Šula, 1996). The activity of the CA in macropterous females is suppresssed for, at least, two weeks after adult ecdysis. This is indicated by the absence of vitellogenins in hemolymph of 14 days old macropterous females and by about two weeks longer pre-oviposition period in macropterous compared to brachypterous females (Socha \& Šula, 1996). Macropterous females exhibit a low feeding activity during 2-3 days after adult ecdysis and thereafter the feeding activity is negligible for at least two weeks (Socha et al., 1998; Šula et al., 1998). Thus it might be assumed that the endocrine mechanism underlying the reproductive arrest in long-day macropterous females is 
similar to that in the starved long-day brachypterous females. However, the macropterous females are not completely starving and, therefore, a lack of food stimulation may not be the primary cause of the CA suppression.

The chemical identity of the hemipteran juvenile hormone $(\mathrm{JH})$ has still remained unknown (Davey, 1997; Hodková et al., 2001) and thus the activity of the CA cannot be determined directly by the measurements of $\mathrm{JH}$ titre in hemolymph. The denervated CA always stimulated ovarian maturation, while the complexes without CA had no effect (Hodková, 1975, 1977a, 1982). The level of reproductive performance stimulated by the implanted BR-SG-CC-CA can, therefore, indicate the level of inhibition of the CA by the attached BR-SG. We used reciprocal transplantations of neuroendocrine complexes between macropterous and brachypterous females of $P$. apterus to determine whether the suppression of the $\mathrm{CA}$ in macropterous females is attributable to the diapause-like refractoriness of the BR-SG-CC-CA or an unsufficient stimulation from the internal milieu.

\section{MATERIAL AND METHODS}

\section{Experimental insects}

The laboratory stock cultures of Pyrrhocoris apterus (L.) were established from the bugs collected at České Budějovice (Czech Republic, $49^{\circ} \mathrm{N}$ ). Both brachypterous and macropterous cultures (Socha et al., 1997, 1998) were reared on linden seeds and water ad libitum at constant temperature of $26 \pm 1{ }^{\circ} \mathrm{C}$ and under a long-day photoperiod of $18 \mathrm{~L}: 6 \mathrm{D}$ allowing continuous breeding. Diapause bugs were obtained from the cultures reared from egg to adult under a short-day photoperiod of $12 \mathrm{~L}: 12 \mathrm{D}$ and temperature $26 \pm 1{ }^{\circ} \mathrm{C}$. Larvae and adults from all cultures were kept in 0.51 glass jars in mass culture (approximately 30-40 specimens per jar), and food and water were replenished twice a week. Adult females were used in all experiments.

\section{Surgical procedures}

Females destined for surgical manipulations, including donors and recipients, were deprived of food within several hours after adult ecdysis and operated two days later. All surgical manipulations, including nerve transection, allatectomy and implantation of the neuroendocrine complex, were performed through the neck membrane incision under Ringer insect saline. The CA was denervated by transection of the nervi allati together with aorta. Neck membrane was cut in the sham-operated females. Females were allatectomized immediately before the implantation of the neuroendocrine complex of brain-corpora cardiacacorpus allatum with attached suboesophageal ganglion and part of aorta (BR-SG-CC-CA). Three groups of females were used as donors and recipients: (1) long-day macropterous females; (2) long-day brachypterous females; (3) diapause short-day bra-

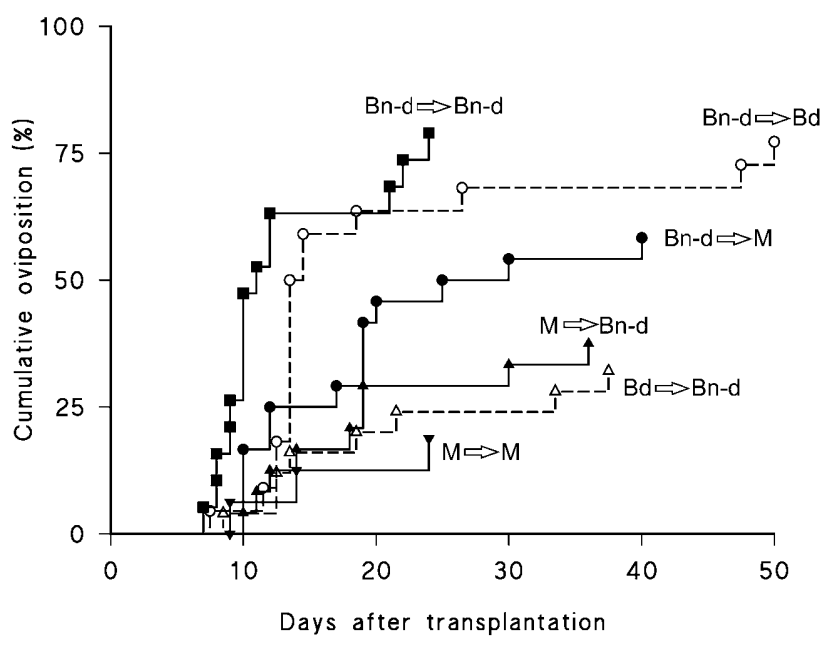

Fig. 1. Transplantations of neuroendocrine complexes between macropterous and brachypterous females of P. apterus. Neuroendocrine complex of brain-corpora cardiaca-corpus allatum with attached suboesophageal ganglion and part of aorta (BR-SG-CC-CA) was transplantated from/to brachypterous non-diapause (Bn-d), brachypterous diapause (Bd), and macropterous (M) females. Donor is indicated by the abbreviation to the left of the arrow, recipient is indicated by the abbreviation to the right of the arrow. Donors and recipients were starved for two days after adult ecdysis, recipients were allatectomized immediately before transplantation. See also Table 1.

chypterous females. All recipients were given food immediately after operation. One week later, females were isolated and thereafter kept individually in Petri dishes $(\varnothing=6 \mathrm{~cm})$. The endocrine activity of the implanted neuroendocrine complex was estimated by the reproductive output of recipients during the 50 days of the experimental period. The length of pre-oviposition period (interval in days between the day of operation and first oviposition) was measured in all experimental groups.

\section{Measurements of corpus allatum and terminal oocytes}

All micrometrical measurements were carried out with an ocular micrometer adapted to stereomicroscope. Bürker chamber of the depth of $0.01 \mathrm{~mm}$ was used for the measurement of the CA volume that was calculated according to the formula: $\pi \mathrm{a} \mathrm{b} \mathrm{d} \mathrm{(a} \mathrm{and} \mathrm{b}=$ radii, $\mathrm{d}=$ depth of Bürker chamber). Terminal oocyte length was measured in 2-3 ovarioles chosen at random in the ovary pair, and the values were averaged. For each experimental group, organs were measured in, at least, 10 animals.

\section{Statistical analyses}

Statistical evaluation of results was made by means of the software GraphPad Prism, version 4.0 (San Diego, CA, USA).

TABLE 1. Transplantations of neuroendocrine complexes between macropterous and brachypterous females of P. apterus

\begin{tabular}{cllrrr}
\hline \multirow{2}{*}{ Experiment } & \multirow{2}{*}{ Donor } & Recipient & $n$ & \multicolumn{2}{c}{ Ovipositing females } \\
\cline { 4 - 6 } & & & Number & $\%^{1}$ \\
\hline A & & Macropterous & 16 & 3 & $18.8 \mathrm{a}$ \\
B & & Brachypterous non-diapause & 24 & 9 & $37.5 \mathrm{ab}$ \\
C & Macropterous & Macropterous & 24 & 14 & $58.3 \mathrm{bc}$ \\
D & Brachypterous non-diapause & Brachypterous non-diapause & 19 & 15 & $78.9 \mathrm{c}$ \\
E & Brachypterous diapause & Brachypterous non-diapause ${ }^{2}$ & 22 & 17 & $77.3 \mathrm{c}$ \\
F & B & 25 & 8 & $32.0 \mathrm{a}$ \\
\hline
\end{tabular}

${ }^{1}$ Fisher test, significantly different values $(\mathrm{P}<0.05)$ are indicated by different letters; ${ }^{2}$ Data partly from Hodková $(1992)$. 


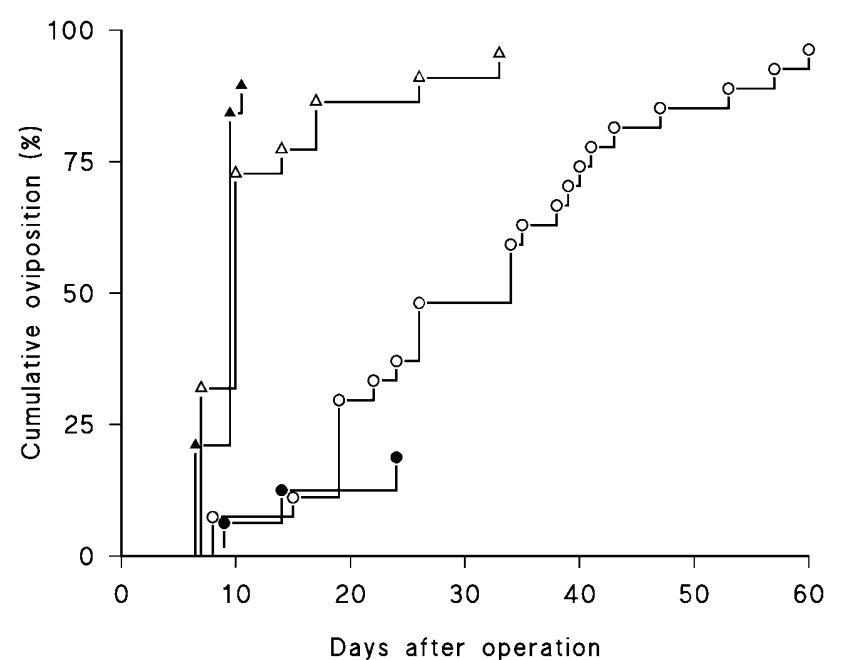

Fig. 2. Denervation of the corpus allatum in macropterous females of P. apterus. Open circles - non-operated $(n=27)$; open triangles - sham-operated $(n=21)$; closed circles - corpus allatum removed, neuroendocrine complex of brain - suboesophageal ganglion - corpora cardiaca - corpus allatum implanted ( $n=16$, see also Table 1A and Fig. 1); closed triangles - corpus allatum denervated $(n=19)$.

\section{RESULTS}

\section{Transplantation of "starving" neuroendocrine complexes into feeding females}

The neuroendocrine complexes of BR-SG-CC-CA were transplanted between macropterous and brachypterous females, both kept under long days. The results were compared with those obtained from earlier transplantations between diapause and non-diapause brachypterous females (Hodková, 1992). The BR-SG-CC-CA from long-day brachypterous females stimulated reproduction in more than three quarters of long-day brachypterous females within 24 days, while only less than one-third of recipients was stimulated by the complex from macropterous females within the same period (Fig. 1) and only slightly more than one-third of recipients started oviposition within the 50-day experiment (Table 1, B vs. D). A similar difference was observed after the transplantation of complexes into long-day macropterous females (Fig. 1, Table 1, A vs. C). The activity of the BR-SG-CC-CA from macropterous females was similar to the activity of the complex from short-day brachypterous females (Fig. 1, Table 1, B vs. F, Hodková, 1992). The results indicate that the "macropterism", similar to the diapause, is associated with a considerably lower responsiveness of the BRSG-CC-CA to humoral stimulation by feeding compared to the responsiveness of the "starving" complex from brachypterous non-diapause females.

On the other hand, there was no clear difference among effects of the internal milieu of feeding long-day macropterous, long-day brachypterous and diapause brachypterous females. Although the proportion of ovipositing females was slightly higher in brachypterous than in macropterous recipients, the difference was not significant (Fig. 1, Table 1, C vs. D vs. E). It appears, therefore, that

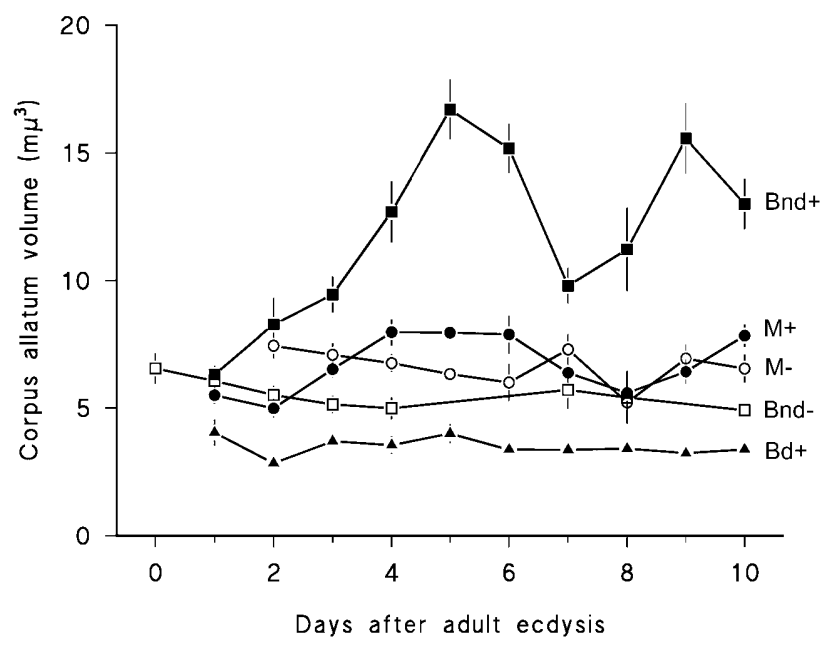

Fig. 3. Changes in size of the corpus allatum in macropterous and brachypterous females of $P$. apterus. Closed circles - macropterous females, food available $(\mathrm{M}+)$; open circles - macropterous females, food absent (M-); closed squares brachypterous non-diapause females, food available (Bnd+); open squares - brachypterous non-diapause females, food absent (Bnd-); triangles - brachypterous diapause females, food available $(\mathrm{Bd}+)$. Each point represents an average of at least 10 individuals \pm SEM. ANOVA, Newman-Keuls Multiple Comparison Test: $\mathrm{M}+$ vs. $\mathrm{M}-$ vs. $\mathrm{Bnd}-$ (n.s., $P>0.05$ ); $\mathrm{Bd}+$ vs. Bnd- $(P<0.05)$; Bd + vs. $\mathrm{M}+$, $\mathrm{M}-$, $\mathrm{Bnd}+(P<0.001)$; Bnd + vs. $\mathrm{M}+, \mathrm{M}-$, Bnd- $(P<0.001)$.

the reproductive activity depends more on the origin of the transplanted neuroendocrine complex than on the internal milieu of recipients.

\section{Denervation of the corpus allatum}

To determine whether the low activity of the BR-SG$\mathrm{CC}-\mathrm{CA}$ from the macropterous females is caused by an inhibition of the CA by the attached brain, the nerves between $\mathrm{CC}$ and $\mathrm{CA}$ were cut. The operation resulted in oviposition in $89.5 \%$ of females within 10 days (Fig. 2). The pre-oviposition period $(9.4 \pm 0.3$ days $)$ was significantly shorter $(P<0.0001)$ than the pre-oviposition period of non-operated females $(31 \pm 2.8$ days $)$, but similar to the pre-oviposition period of sham-operated females (11.7 \pm 1.5 days). Thus the injury stimulated reproduction irrespective of whether the CA was connected to the brain or denervated. The results indicate that the brain in situ does not inhibit the CA in injured macropterous females.

However, the proportion of ovipositing females was markedly higher after the denervation of the CA $(89.5 \%$ within 10 days, Fig. 2) than after the implantation of the BR-SG-CC-CA into allatectomized recipients $(18.8 \%$ within 50 days, Fig. 1, Table $1 \mathrm{~A}$ ), although the injury was similar in both groups. This suggests that the $\mathrm{CA}$ is inhibited by the brain within the transplanted BR-SGCC-CA in spite of the injury of recipient.

\section{Size of the corpus allatum}

In brachypterous females, the lower activity of the BR$\mathrm{CC}-\mathrm{CA}$ from diapause vs. starving non-diapause females 


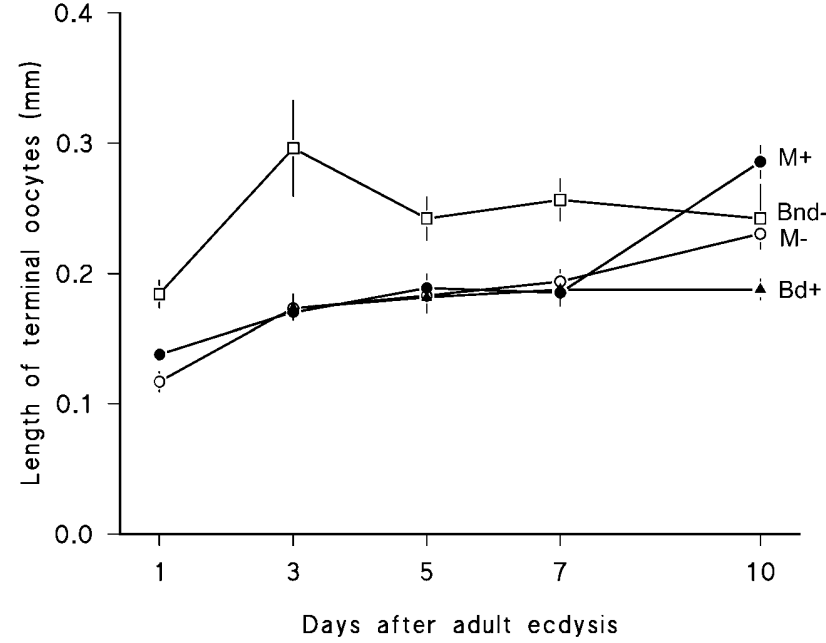

Fig. 4. Changes in size of the terminal oocyte in macropterous and brachypterous females of $P$. apterus. Closed circles - macropterous females, food available $(\mathrm{M}+)$; open circles - macropterous females, food absent $(\mathrm{M}-)$; open squares brachypterous non-diapause females, food absent (Bnd-); triangles - brachypterous diapause females, food available $(\mathrm{Bd}+)$. Each point represents an average of at least 10 individuals \pm SEM. ANOVA, Newman-Keuls Multiple Comparison Test, days 1-7: Bnd- vs. $\mathrm{M}+, \mathrm{M}-, \mathrm{Bd}+(P<0.05)$; $\mathrm{M}+$ vs. $\mathrm{M}-$ vs. $\mathrm{Bd}+$ (n.s., $P>0.05$ ).

(Table 1, D vs. F) was associated with a smaller CA (Fig. 3, Hodková, 1999). Although a similar difference was observed in the activity of the "starving" BR-SG-CC-CA from macropterous vs. brachypterous non-diapause females (Table 1, B vs. D), the size of the CA in macropterous females was not reduced (Fig. 3). In fact, the CA of starving macropterous females was significantly larger than the CA of brachypterous diapause females (Fig. 3), although there was no significant difference in the activity of the BR-SG-CC-CA (Table 1, B vs. F). In contrast to brachypterous non-diapause females, the CA of macropterous females remained small even in the presence of food (Fig. 3).

\section{Size of the terminal oocyte}

Although the CA in macropterous females was larger than the CA in brachypterous diapause females (Fig. 3), the terminal oocytes had similar size for, at least, one week after adult ecdysis and were smaller than the terminal oocytes in starving brachypterous non-diapause females (Fig. 4). In macropterous females, the presence of food had no effect on the size of oocytes during one week after adult ecdysis. In contrast to brachypterous diapause females, the oocytes of macropterous females started to increase in size one week after adult ecdysis. While the oocytes of macropterous females remained in previtellogenic stage for at least the first 10 days of adult life, irrespective of food conditions (Fig. 4), brachypterous non-diapause females oviposited already on the 6th-7th day after adult ecdysis if they had access to food.

\section{DISCUSSION}

Regulation of the corpus allatum activity and ovarian growth in $P$. apterus females

Biosynthesis of juvenile hormone $(\mathrm{JH})$ by the $\mathrm{CA}$ is strongly influenced by brain neuropeptides (Stay, 2000; Gäde \& Hoffmann, 2005), but the role of brain regulators in the morph-specific differences has not yet been studied. The present study on macropterous females of $P$. apterus shows that the gonadotropic activity of the denervated $\mathrm{CA}$ is much higher compared to the $\mathrm{CA}$ of the transplanted BR-SG-CC-CA (Figs 1 and 2, Table 1A), thus indicating the nervous inhibition of the CA within the transplanted neuroendocrine complex. In contrast to the transplanted BR-SG-CC-CA, the gonadotropic activity of the neuroendocrine complex in situ was stimulated by injury in macropterous females also in the case when the nervous connection between the brain and the CA was not interrupted (Fig. 2). It was demonstrated that stresses induced the release of neurosecretory materials affecting the spontaneous nerve activity of the central nervous system (see Raabe, 1982, pp. 173-174). It seems that the stimulating effect of injury is transmitted to the neuroendocrine complex in situ through the intact nervous system, because most transplanted complexes were not stimulated (Table 1A). Therefore, the nervous inhibition of the CA could not be demonstrated with the brain in situ. Interestingly, artificially de-alated, i.e. injured, long-winged females of a cricket, Modicogryllus confirmatus, produced more eggs and consumed more food than intact females of this wing morph (Tanaka, 1993).

The low feeding activity of macropterous females ( ̌́ula et al., 1998) does not seem to be the only cause of the absence of vitellogenesis and oviposition; $58 \%$ of the macropterous recipients oviposited after transplantation of the BR-SG-CC-CA complex from the starving brachypterous females, while the complex from the macropterous females stimulated oviposition in only $18.8 \%$ of macropterous recipients (Table $1 \mathrm{~A}, \mathrm{C}$ ). A similar difference between gonadotropic activities of these complexes was observed after their transplantation into the internal milieu of brachypterous females (Table 1 B, D). Therefore, the low gonadotropic activity of the transplanted "macropterous" BR-SG-CC-CA seems to result mainly from a refractoriness of the neuroendocrine complex to the stimulating internal milieu of feeding females. The internal milieu of macropterous females is sufficiently stimulating to overcome the inhibition of the CA within the non-refractory complex from starving brachypterous females. The gonadotropic activity of the implanted "macropterous" BR-SG-CC-CA was as low as that of the "brachypterous diapause" complex (Table 1 B, F). Thus the "macropterism" and diapause seem to result in similar refractoriness of the neuroendocrine complexes to the stimulating internal milieu. However, macropterous and diapause brachypterous females differ in two important points. (1) The inhibition of the CA in diapause brachypterous females is not removed by injury irrespective of whether the neuroendocrine complex is in situ or trans- 
planted (Hodková, 1976, 1977a, b, 1992). (2) The CA of macropterous females is significantly larger than the CA of brachypterous diapause females (Fig. 3). This indicates that the refractoriness of the "macropterous" neuroendocrine complex is not associated with a diapause-like inhibition of the growth of the CA.

The CA of macropterous females has an intermediate size, similar to the CA of starving brachypterous females. In both groups of females, the activity of the CA is below the threshold for vitellogenesis, but, given the lack of information on the hemipteran $\mathrm{JH}$, the titre of $\mathrm{JH}$ in hemolymph could not be compared. If similar volumes of the CA indicate similar intermediate activities of the CA, it is not clear why the pre-vitellogenic growth of oocytes in macropterous females was delayed when compared to starving brachypterous females. In fact, the size of oocytes during the first 7 days of adult life was, in the former group, similar to that observed in diapause (Fig. 4) or allatectomized non-diapause brachypterous females (Hodková, 1999), where JH was absent. Therefore, it cannot be excluded that, in spite of similar volumes of the $\mathrm{CA}$, the activity of the CA was lower in macropterous than in starving brachypterous females. Alternatively, the high dispersal activity (Socha \& Zemek, 2003), associated with enhanced titre of adipokinetic hormone (Kodrík et al., 2003) may be responsible for the depletion of fat body reserves and, consequently, a delay in the previtellogenic growth of oocytes in macropterous females of $P$. apterus with an intermediate activity of the CA. This suggestion is supported by the most recent study showing that the development of indirect flight muscles in long-day macropterous females of P. apterus is accomplished during the first 5-7 days after adult ecdysis (Socha \& Šula, 2006), when the pre-vitellogenic growth of oocytes is inhibited (Fig. 4). A similar prevalent allocation of the resources to flight muscles (rather than to oocytes) was reported for long-winged females of the crickets, Allonemobius fasciatus and M. confirmatus (Tanaka, 1986, 1993). In macropterous females of $P$. apterus older than 14 days, the histolysis of flight muscles coincides with resumption of food intake, growth of vitellogenic oocytes (Socha \& Šula, 2006) and an increase in the CA size (data not shown). Since the vitellogenin synthesis is under $\mathrm{JH}$ control in $P$. apterus females (Socha et al., 1991), the increase in the CA size in older macropterous females is most probably associated with an increase of the CA activity to or above the threshold for vitellogenesis.

\section{Role of JH in an "oogenesis-walking" syndrome of $\boldsymbol{P}$. apterus females}

The original proposal that the temporal relationship between migratory flight and reproduction, designated the "oogenesis-flight syndrome", is mediated by JH (Johnson, 1969) led to the development of a model suggesting that low or intermediate titre of $\mathrm{JH}$ stimulated migration, whereas reproduction was stimulated at higher titres of JH (Rankin \& Riddiford, 1977). Data from the milkweed bug, Oncopeltus fasciatus, (Rankin \& Riddiford, 1977) and some other flying insects (Rankin et al.,
1986; McNeil et al., 1995; Dingle \& Winchell, 1997) generally support the model.

The trade-off between dispersal capability and reproduction (for review see Zera \& Denno, 1997), including an "oogenesis-flight syndrome" (Johnson, 1969), was originally reported only for flying monomorphic and wing-polymorphic insect species whose all or at least some of the macropterous specimens are capable of flight. However, our recent studies showed that trade-off between dispersal capability and reproduction occurs also in macropterous females of $P$. apterus that changed their dispersal modus from flight to walking only (Socha \& Zemek, 2003; Socha, 2004). We have also demonstrated that enhanced walking and dispersal activities (Socha \& Zemek, 2000, 2003) of recent flightless macropters of $P$. apterus are ensured by the same physiological energymobilization system that was originally used by their ancestors for flight (Socha \& Kodrík, 1999, Kodrík et al., 2003). All the above data indicate that the original "oogenesis-flight" syndrome has been secondarily modified to the "oogenesis-walking" syndrome in macropterous females of $P$. apterus during the evolution of flightlessness (Socha, 2004). An occurrence of this phenomenon is important from both the ecological and evolutionary points of view.

According to a widely accepted model, the titre of $\mathrm{JH}$ plays a key role in morph-specific differences in development and reproduction of wing-polymorphic species (reviewed in Zera, 2004). Earlier data on adult morphs of a wing-polymorphic cricket, Gryllus firmus, indicated that an elevated JH titre may be at least partly responsible for the increased early fecundity of flightless females (Cisper et al., 2000). Recently, a morph-specific daily cycle in the haemolymph $\mathrm{JH}$ titre, starting on day 5 of adulthood, has been revealed in G. firmus (Zhao \& Zera, 2004a). A morph-specific daily cycle in the in vitro rate of JH biosynthesis was tightly correlated with the haemolymph $\mathrm{JH}$ titre on days 5-7 of adulthood (Zhao \& Zera, 2004 b). However, a relatively long (two weeks) period of reproductive arrest and oviposition delay in macropterous females of $P$. apterus associated with low or intermediate activity of the CA and enhanced dispersal activity resembles the "oogenesis-flight" syndrome rather than the decreased early fecundity found in the wing-polymorphic crickets (Tanaka, 1993, 1994; Zera et al., 1993; Cisper et al., 2000).

In conclusion, we showed that (1) the CA of long-day macropterous females of $P$. apterus is of intermediate size between that of the long-day (non-diapause) and short-day (diapause) brachypterous females, similar to the CA of the non-diapause brachypterous females deprived of food, and (2) reproduction arrest in long-day macropterous females results mainly from a refractoriness of the neuroendocrine complex to the stimulating internal milieu, similar to the short-day (diapause) brachypterous females. Thus the suppression of the CA activity in long-day macropterous females seems to result from a combination of the diapause-like refractoriness of the neuroendocrine complex with the starvation-like inhibi- 
tion of the CA growth. A search for the factors playing the main role in terminating the $\mathrm{CA}$ inhibition and reproduction arrest in macropterous females of $P$. apterus is a topic of the current study.

ACKNOWLEDGEMENTS. This study was supported by grants No 206/03/001 (RS) and 206/05/2222 (MH) from the Grant Agency of the Czech Republic and Z50070508 of the Academy of Sciences of the Czech Republic. The authors thank J. Mikešová and D. Rienesslová for their technical assistance.

\section{REFERENCES}

Cisper G., Zera A.J. \& Borts D.W. 2000: Juvenile hormone titer and morph-specific reproduction in the wingpolymorphic cricket, Gryllus firmus. J. Insect Physiol. 46: 585-596.

DAVEY K.G. 1997: Hormonal controls on reproduction in female Heteroptera. Arch. Insect Biochem. Physiol. 35: 443-453.

Dingle H. \& Winchell R. 1997: Juvenile hormone as a mediator of plasticity in insect life histories. Arch. Insect Biochem. Physiol. 35: 359-373.

GädE G. \& HoffmanN K.-H. 2005: Neuropeptides regulating development and reproduction in insects. Physiol. Entomol. 30: $103-121$.

HoDEK I. 1968: Diapause in females of Pyrrhocoris apterus L. (Heteroptera). Acta Entomol. Bohemoslov. 65: 422-435.

Hodková M. 1975: Regulation of the Corpus Allatum Activity in Pyrrhocoris apterus (Hemiptera). PhD Thesis, Czechoslovak Academy of Sciences, Prague, 138 pp. [in Czech].

Hodková M. 1976: Nervous inhibition of corpora allata by photoperiod in Pyrrhocoris apterus. Nature 263: 521-523.

HodKovÁ M. 1977a: Function of the neuroendocrine complex in diapausing Pyrrhocoris apterus females. J. Insect Physiol. 23: 23-28.

HodkovÁ M. 1977b: Nervous pathways in photoperiodic regulation of reproduction in females of Pyrrhocoris apterus (Hemiptera). Acta Entomol. Bohemoslov. 74: 353-361.

HodKovÁ M. 1977c: Size and gonadotropic activity of corpus allatum after different surgical treatments in Pyrrhocoris apterus females (Heteroptera). Věst. Čs. Spol. Zool. 41: 8-14.

Hodkové M. 1979: Hormonal and nervous inhibition of reproduction by brain in diapausing females of Pyrrhocoris apterus L. (Hemiptera). Zool. Jb. Physiol. 83: 126-136.

HodKoví M. 1982: Interaction of feeding and photoperiod in regulation of the corpus allatum activity in females of Pyrrhocoris apterus L. (Hemiptera). Zool. Jb. Physiol. 86: 477-488.

HoDKovÁ M. 1992: Storage of the photoperiodic "information" within the implanted neuroendocrine complexes of the linden bug, Pyrrhocoris apterus (L.) (Heteroptera). J. Insect Physiol. 38: $357-363$.

HodkovÁ M. 1999: Regulation of diapause and reproduction in Pyrrhocoris apterus (L.) (Heteroptera) - neuroendocrine outputs (mini-review). Entomol. Sci. 2: 563-566.

Hodková M., OKuda T. \& Wagner R.M. 2001: Regulation of corpora allata in females of Pyrrhocoris apterus (Heteroptera) (a mini-review). In Vitro Cell Dev. Biol. Anim. 37: 560-563.

HoNĚK A. 1985: Ecophysiological differences between brachypterous and macropterous morphs in Pyrrhocoris apterus (Heteroptera, Pyrrhocoridae). Acta Entomol. Bohemoslov. 82: $347-354$

Johnson B. 1969: Migration and Dispersal of Insects by Flight. Methuen, London, 763 pp.

Kodrík D., Socha R. \& Syrová Z. 2003: Developmental and diel changes of adipokinetic hormone in CNS and haemo- lymph of the flightless wing-polymorphic bug, Pyrrhocoris apterus (L.). J. Insect Physiol. 49: 53-61.

McNeil J.N., Cusson M., Delisle J., Orchard I. \& Tobe S.S. 1995. Physiological integration of migration in Lepidoptera. In Drake V.A. \& Gatehouse A.G. (eds): Insect Migration: Physical Factors and Physiological Mechanisms. Cambridge University Press, Cambridge, pp. 193-242.

RAABE M. 1982: Insect Neurohormones. Plenum Press, New York, $352 \mathrm{pp}$.

RANKIN M.A. \& RIDDIFORD L.M. 1977: Hormonal control of migratory flight in Oncopeltus fasciatus: the effects of the corpus cardiacum, corpus allatum and starvation on migration and reproduction. Gen. Comp. Endocrinol. 33: 309-321.

Rankin M.A., McAnelly M.L. \& Bodenhamer J.E. 1986: The oogenesis-flight syndrome revisited. In Danthanarayana E. (ed.): Insect Flight: Dispersal and Migration. SpringerVerlag, Berlin, pp. 27-48.

SAUNDERS D.S. 1983: A diapause induction-termination asymmetry in the photoperiodic responses of the linden bug, Pyrrhocoris apterus and an effect of near-critical photoperiods on development. J. Insect Physiol. 29: 399-405.

SochA R. 1993: Pyrrhocoris apterus (Heteroptera) - an experimental model species: a review. Eur. J. Entomol. 90: 241-286.

SochA R. 2004: Decreased mating propensity of macropterous morph in a flightless wing-polymorphic insect, Pyrrhocoris apterus (L.) (Heteroptera). Eur. J. Entomol. 101: 539-545.

Socha R. \& KodRíK D. 1999: Differences in adipokinetic responses of Pyrrhocoris apterus (Heteroptera) in relation to wing dimorphism and diapause. Physiol. Entomol. 24: 278-284.

Socha R. \& Šula J. 1996: Differences in haemolymph proteins in relation to diapause and wing dimorphism in Pyrrhocoris apterus (L.) (Heteroptera: Pyrrhocoridae). J. Comp. Physiol. (B) 166: 382-387.

Socha R. \& Šula J. 2006: Flight muscles polymorphism in a flightless bug, Pyrrhocoris apterus (L.): developmental pattern, biochemical profile and endocrine control. J. Insect Physiol. 52: 231-239.

Socha R. \& Zemek R. 2000: Locomotor activity in adult Pyrrhocoris apterus (Heteroptera) in relation to sex, physiological status and wing dimorphism. Physiol. Entomol. 25: 383-389.

SOCHA R. \& ZEMEK R. 2003: Wing morph-related differences in the walking pattern and dispersal in a flightless bug, Pyrrhocoris apterus (L.) (Heteroptera). Oikos 100: 35-43.

Socha R., Šula J., Kodrík D. \& Gelbič I. 1991: Hormonal control of vitellogenin synthesis in Pyrrhocoris apterus (L.) (Heteroptera). J. Insect Physiol. 37: 805-816.

Socha R., Šula J. \& ZemeK R. 1997: Feeding, drinking and digestive enzyme activities in long- and short-day females of Pyrrhocoris apterus (Heteroptera). Physiol. Entomol. 22: 161-169.

Socha R., ŠUla J. \& ZEMEK R. 1998: Feeding behaviour, digestive physiology and lipid content in macropterous females of Pyrrhocoris apterus (L.) (Heteroptera: Pyrrhocoridae). Physiol. Entomol. 23: 91-96.

Stay B. 2000: A review of the role of neurosecretion in the control of juvenile hormone synthesis: a tribute to Berta Scharrer. Insect Biochem. Molec. Biol. 30: 653-662.

Šula J., Socha R. \& Zemek R. 1998: Wing morph-related physiological differences in adults of temperate population of Pyrrhocoris apterus (L.) (Heteroptera: Pyrrhocoridae). Сomp. Biochem. Physiol. (A) 121: 365-373.

TANAKA S. 1986: De-alation, flight muscle histolysis and oocyte development in the striped ground cricket, Allonemobius fasciatus. Physiol. Entomol. 11: 453-458. 
TANAKA S. 1993: Allocation of resources to egg production and flight muscle development in a wing dimorphic cricket, Modicogryllus confirmatus. J. Insect Physiol. 39: 493-498.

TANAKA S. 1994: Endocrine control of ovarian development and flight muscle histolysis in a wing dimorphic cricket, Modicogryllus confirmatus. J. Insect Physiol. 40: 483-490.

ZERA A.J. 2004: The endocrine regulation of wing polymorphism in insects: state of the art, recent surprises and future directions. Integr. Comp. Biol. 46: 607-616.

Zera A.J. \& DenNo R.F. 1997: Physiology and ecology of dispersal polymorphism in insects. Annu. Rev. Entomol. 42: 207-231.
Zera A.J., Borcher C.A. \& Gaines S.B. 1993: Juvenile hormone degradation in adult wing morphs of the cricket, Gryllus rubens. J. Insect Physiol. 39: 845-856.

Zhao Z. \& Zera A.J. 2004a: A morph-specific daily cycle in the rate of JH biosynthesis underlies a morph-specific daily cycle in the hemolymph JH titer in a wing-polymorphic cricket. $J$. Insect Physiol. 50: 960-973.

Zhao Z. \& Zera A.J. 2004b: The haemolymph JH titer exhibits a large-amplitude, morph-dependent, diurnal cycle in the wing-polymorphic cricket, Gryllus firmus. J. Insect Physiol. 50: $93-102$.

Received December 7, 2005; revised and accepted February 28, 2006 